\title{
A Novel Approach to Reduce Packet Loss in OBS Networks
}

\author{
Amit Gupta ${ }^{1}$, Harbhajan Singh ${ }^{2}$, Jagdish Kumar ${ }^{3}$ \\ ${ }^{1}$ Deptt. of Electronics and Communication Engg, PTU, Jalandhar, India. \\ ${ }^{2}$ Deptt. of Electronics and Communication Engg, SSIET, Derabassi (Punjab), India \\ ${ }^{3}$ Deptt. of Electrical Engg, PEC University of Technology, Chandigarh (UT), India
}

\begin{abstract}
In this paper, we propose an efficient deflection routing method to improve the burst loss and throughput performance in Optical Burst Switched (OBS) networks. We show that the proposed scheme gives significant reduction in burst loss rate and considerable improvement in throughput percentage as compared to the conventional technique for optical networks. To improve the burst loss performance, the proposed congestion based algorithm, dynamically determines the congestion level for apposite flow control. The burst sending rate is adjusted by deflecting the burst according to the traffic condition in a network. Ultimately, the proposed scheme achieves considerable reduction in the burst loss rate and high throughput while maintaining acceptable delay and fairness.
\end{abstract}

Index Terms - Optical Burst Switching (OBS); Congestion Control (CC); Burst Loss Rate (BLR)

\section{INTRODUCTION}

Optical burst switching (OBS) is emerging as a new optical switching paradigm to efficiently support the massive demand of higher transmission rates and faster switching technologies. OBS is a wavelength division multiplexing (WDM) technique that maintains the merits of both, optical packet switching and optical circuit switching [1]. In OBS, an ingress node assembles the internet protocol (IP) packets from various traffic sources. Packets destined to the same egress edge node are aggregated into a single data burst which is transmitted entirely in optical domain [2]. The burst control packet $(\mathrm{BCP})$ with the information on the length and arrival time of the data burst is sent in advance. The BCP is transmitted from an edge node to its destination node followed by the data burst (DB) after a specified time interval called offset time (OT). The offset time estimation requires the precise number of hops from source to destination [3]. OBS experiences similar flexibility as optical packet switching to the variation in traffic. But, OBS has some unsolved technical issues, which include bandwidth reservation schemes, burst scheduling, burst contention, burst assembly and the performance evaluation of these interdependent approaches.

Congestion, the main cause of contention in OBS networks, is a state which affects the performance because of the saturation in network resources. The contention occurs when multiple bursts intend for the same outgoing channel simultaneously in a switch. To make the optical network capable of handling enormous internet applications, there is an acute need to deal with network congestion in OBS network. The most important concern of OBS networks is the packet loss due to burst contention problem. The main contention resolution schemes that have been proposed are: the optical buffering technology using the fiber delay line, wavelength conversion and deflection routing [4]. The first two techniques, being very sophisticated and immature for optical hardware devices, divert more attention towards deflection routing technique [5]. The basic idea of deflection routing technique is to use the other alternate unused paths to avoid the contention. The deflection routing can reduce the packet loss rate to minimum at low loads. However, at higher loads the packet loss rate is still significant. Thus, to improve the packet loss performance, a more refined, flexible and fool-proof method of alternate routing should be employed.

So far, several approaches have been proposed for highperformance switching that can more effectively exploit the capabilities of fiber-optic transmission systems as also to resolve packet loss rate due to contention. Shamim Reza et al proposed a scheme to minimize burst loss rate in the network by using wavelength converters and by increasing the number of channel wavelengths [6]. In [7], burst loss information received from all core nodes is responsible for adjusting the burst sending rate by the ingress node. A TCP congestion control scheme for OBS networks was proposed by Wang et al [8]. In this scheme, the egress node acknowledges the ingress node, whenever it receives a burst. Based on received acknowledgement packets from egress node, the ingress node adjusts the burst sending rate. However, the above schemes may activate improperly because most of the burst loss occurs randomly. Farahmand et al in [9] suggested, the feedback message from each core node should inform each ingress node to adjust its burst sending rate based on control information in the feedback message. But, this complex function unnecessarily burdens each core node, as each core node has to inform all the ingress nodes whether they should increase or decrease the burst sending rate. The deflection routing studies, which use an alternative link computing method, consider either random selection of the output port; demonstrated by $\mathrm{Y}$. Chen et al in [10] or to next shortest path to destination by Zalesky et al [11]. Coutelen et al 
proposed new deflection routing strategies based on different alternative path computing methods with a burst dropping anticipated and features like least loaded node, with U-turn and load balancing [12]. Hui et al in [13] proposed an adaptive provisioning mechanism based on reinforcement-learning principles, which determines the amount of bandwidth to provision to each per hop behavior aggregate. However, due to the bursty and unpredictable nature of traffic, this adaptive provisioning approach is not sufficient to control the congestion, and additional reactive controls may be necessary in the network. More recently, Garg et al in [14] proposed an efficient deflection routing scheme for buffer-less OBS networks to reduce blocking probability caused by offset time and burst contention. This deflection routing method demonstrated in [14] will be taken as 'the conventional' scheme in the context of this research.

All these techniques have been thoroughly investigated and it is found that most of the schemes are restricted only to some specific application or network, rather than a flexible solution which controls the network traffic volume properly. Existing conventional schemes discussed in the literature cannot be considered to be completely preventive for reducing burst dropping as instead of avoiding the early destruction, these techniques adjusts the burst sending rate after the occurrence of contention or burst loss. Thus, to improve the burst loss performance of the optical network, we need to develop an efficient deflection routing contention-control method in which the network overloads are minimized or eliminated to maximize throughput rate.

In this paper, we propose a new congestion based deflection routing (CBDR) algorithm to reduce the packet loss in OBS networks. To reduce the burst loss, contending burst is deflected to another least congested node or in the worst case, back to the previous node after receiving the congestion information from the subsequent node in the path. Burst dropping is avoided by varying the predefined offset time between the burst and the BCP. The proposed technique is investigated through simulations. The proposed technique is tested on variety of performance scenarios to analyze its feasibility as an acceptable solution. Also, we consider the fairness performance between competing traffic flows. Furthermore, we consider performance optimization which is related to the rate-decision problem. Then, we show that the proposed scheme gives significant reduction in the burst loss rate and high throughput performance as compared to the conventional technique.

The rest of the paper is organized as follows:

In section II, we describe the proposed algorithm. In section III, we evaluate the proposed scheme through simulations. Finally, we conclude this paper in section IV.

\section{PROPOSED METHOD}

To overcome the restrictions of previously discussed contention resolution techniques, we propose the congestion based deflection routing (CBDR) algorithm. In this technique, congested core routers need to send some form of feedback information about various traffic flows to previous core nodes and finally to ingress edge routers in the network. So that ingress edge node can deflect the existing burst accordingly and adjust further burst sending rate into the network according to the available bandwidth in the network. In an OBS network, the edge routers have all the capability and intelligence while the core routers have effortless cut-through switching task, to use the feedback information from network to control the congestion problem.

In the proposed contention resolution scheme, each ingress node adjusts its burst sending rate based on the congestion information received from the network.

\section{Algorithm:}

Step 1. Each core node $n_{i}$ sends a feedback message containing congestion level information to previous node $n_{i-1}$ and further to all accessible ingress nodes over the backward path every $T$ units of time. The feedback message is sent on a separate control channel which is dedicated to carry the control information in OBS networks.

Step 2. When the core node $\left(n_{i-1}\right)$ receives the feedback message and if there is no congestion in the network the burst is sent on the predefined path. Subsequently the ingress node will continue sending the burst at the predefined burst sending rate and offset time.

Step 3. However if congestion is detected, core node finds the least congested neighboring node(LCN) to find the alternate forward path, for deflecting the forthcoming burst by sending $\operatorname{INF}_{(I, E)}$ and receiving $\mathrm{CAK}_{(I, E)}$ from all the neighboring nodes .

$\mathrm{INF}_{(\mathrm{I}, \mathrm{E})} \quad$ : Message sent by node $n_{i-1}$ to all neighboring nodes.

$$
\begin{aligned}
\mathrm{CAK}_{(I, E)}: \quad \text { Number of Congestion } \\
\text { Acknowledgement messages (CAK) } \\
\text { received by } n_{i-1} \text { from all } \\
\text { neighbouring nodes on path ingress }
\end{aligned}
$$$$
\text { to egress node }(I, E)
$$

$$
\mathrm{LCN} \leftarrow \operatorname{Min}\left(\mathrm{CAK}_{(I, E)}\right)
$$

Step 4. If the least congested node is found the burst is deflected to that particular core node,

Otherwise, if all the neighboring nodes are equally congested or if neighboring nodes are not available at 
all for deflection, then the burst is sent back to the previous core node $\left(n_{i-2}\right)$ instead of dropping,following the same backward path. Go to Step 3 .

Step 5. The feedback message sent to the ingress node in the step 1 is used to adjust the burst sending rate. We assume that each ingress node can send bursts up to $S_{\max }$ to achieve fairness among all the flows.

\begin{tabular}{|c|c|c|}
\hline & $\mathrm{R}(\mathrm{I}, \mathrm{E})$ & : Current burst sending rate of flow \\
\hline & $\alpha$ & : Increase constant for a single unit \\
\hline & $\beta$ & : Burst rate increase constant. \\
\hline & $\mathrm{H}$ & otal number of hops betwe \\
\hline
\end{tabular}

$$
\mathrm{R}(\mathrm{I}, \mathrm{E}) \leftarrow \mathrm{R}(\mathrm{I}, \mathrm{E}) \times \beta
$$$$
\mathrm{R}(\mathrm{I}, \mathrm{E}) \leftarrow \min \left\{\mathrm{R}(\mathrm{I}, \mathrm{E})+(\alpha \times \mathrm{H}), \mathrm{S}_{\max }\right\}
$$

Step 6. The feedback message sent to the ingress node in the step1 is also used to vary the offset to an appropriate value. Then, subsequent bursts will be sent as per the new amended offset time according to the congestion level in the network.

$$
\begin{array}{ll}
\text { OT } & \text { : Current Offset time } \\
\gamma & \text { : offset change rate. }
\end{array}
$$

m : an integer used to vary $\gamma$

As the congestion level increases/decreases the value of OT will increase/decrease accordingly to adjust the burst sending rate by ingress node.

$\mathrm{OT} \leftarrow \mathrm{OT} \times \gamma^{\mathrm{m}}$

\section{RESULTS \& DISCUSSIONS}

In this section, we evaluate the performance of CBDR scheme presented in the previous section. We then compare the performance of CBDR with the popular shortest path routing scheme. In order to assess the performance of the CBDR technique in an optical network environment and compare it to that of the the conventional deflection routing method, we have developed a simulation model based on the NS-2 platform. The simulation was conducted on the 14 node NSFNET backbone network topology, where all links were assumed to be bi-directional employing a single wavelength per direction. There is no FDL buffering or wavelength conversion at any core node. The link transmission rate is $10 \mathrm{~Gb} / \mathrm{s}$. Length of each arriving packet is taken as $10 \mathrm{~kb}$.

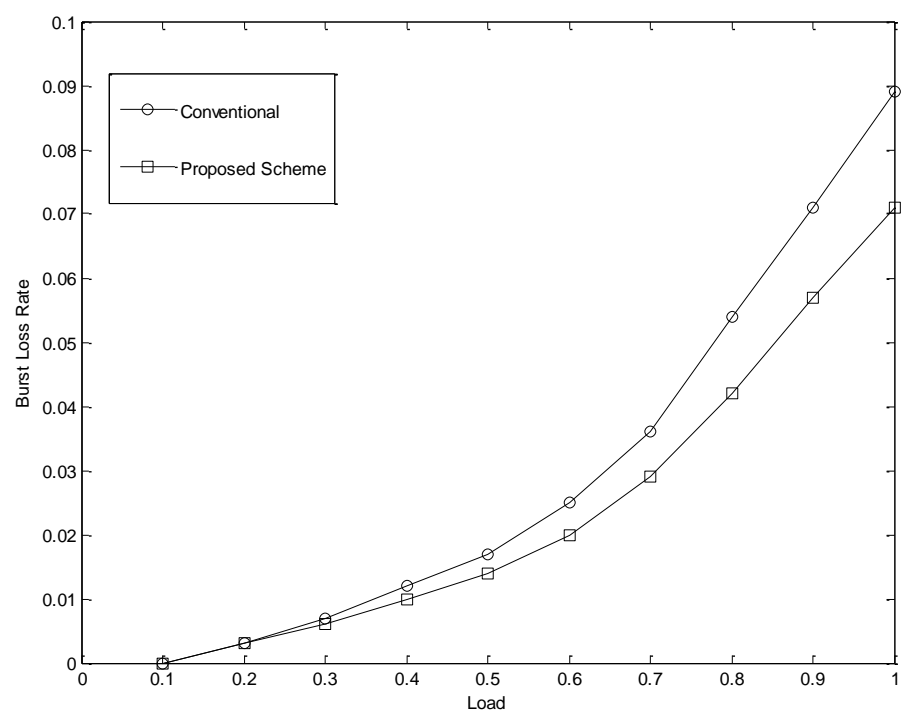

Figure 1: Variation in Burst Loss Rate with respect to Load

In Fig.1, we compared the propose scheme with the conventional one and it is seen that the reduction in burst loss rate up to $14 \%$ is observed with proposed scheme at higher loads. As seen in the figure at low values of load; the burst loss rate varies gradually for both the schemes, but at higher values; it varies abruptly and still more improvement in burst loss rate is achieved in case of proposed scheme. It is examined that overall burst loss rate is high in the conventional technique and low in our propose scheme as expected. 
The improvement in the performance of the proposed approach is due to the fact that various parameters control the contention problem and thus the burst loss reduction in optical networks. In CBDR, the congestion information is sent back to the preceded nodes to take appropriate flow control mechanism, and the same is controlled by finding the least congested node and by varying the offset time at the ingress node. By considering all these constraints in proposed CBDR scheme, a superior method to avoid burst dropping and improving burst loss rate is established.

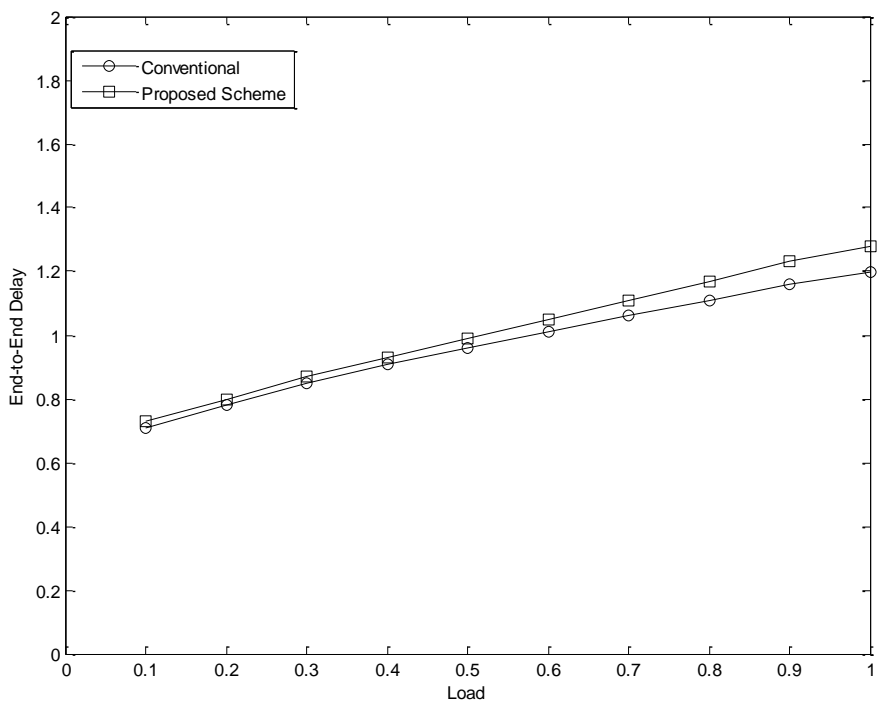

Figure 2: Variation in End-to-End Delay (ms) with respect to Load

Fig. 2 shows end-to-end delay in burst transmission from ingress node to egress node. It is seen that the endto-end delay is high in the proposed scheme, due to deflection felt at many core nodes in case of proposed CBDR algorithm as compared to the conventional technique. It is found that at lower values of load (up to 0.4 ), both schemes observe same gradual gradient, but as the load increases the delay rises with the increase in load. The congestion information, finding of least congested node and coming back to previous node to avoid burst dropping are the major reasons for the higher values of average number of hops travelled and hence the rise in delay is observed. But the delay so observed is comparable to most of the conventional schemes and is in the acceptable limits. The higher value of delay came up with lowering the most important burst drop ratio.

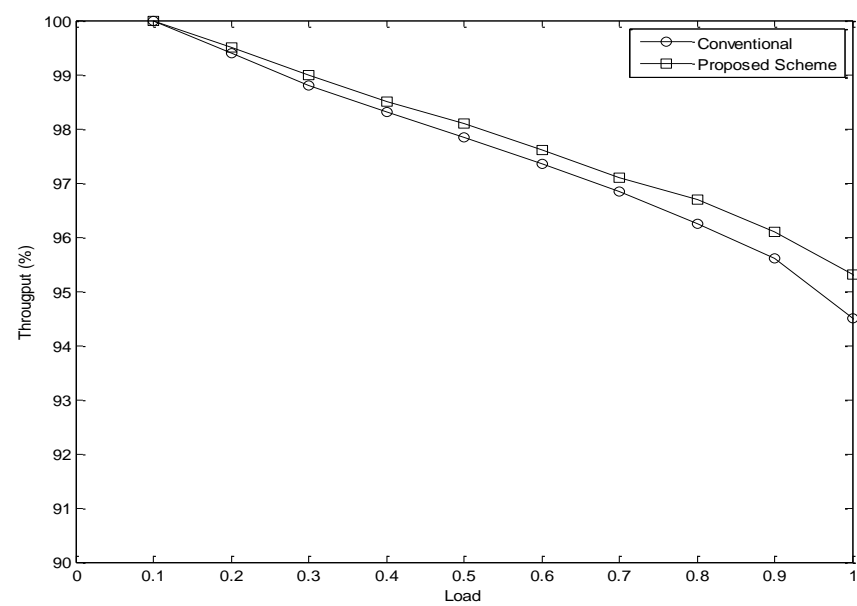

Figure 3: Variation in Throughput with respect to Load 
We investigated the throughput performance of the proposed congestion based technique and the conventional scheme. Fig 3 shows the throughput achieved by CBDR method compared to the conventional for various values of traffic load in OBS networks. At lower values of load the throughput value is almost $100 \%$ because of the well known reason of lower traffic load in the network. But as the load increases, the throughput performance degrades (up to
96\%) due to heavy traffic load for both the schemes under consideration. The throughput achieved by CBDR compared to popular technique is calculated and it is observed that the average throughput achieved by proposed scheme improves marginally because of the efficient deflection routing and lower burst drop ratio. Thus, we know that at all loads; our scheme maintains slightly better throughput performance than the conventional scheme.

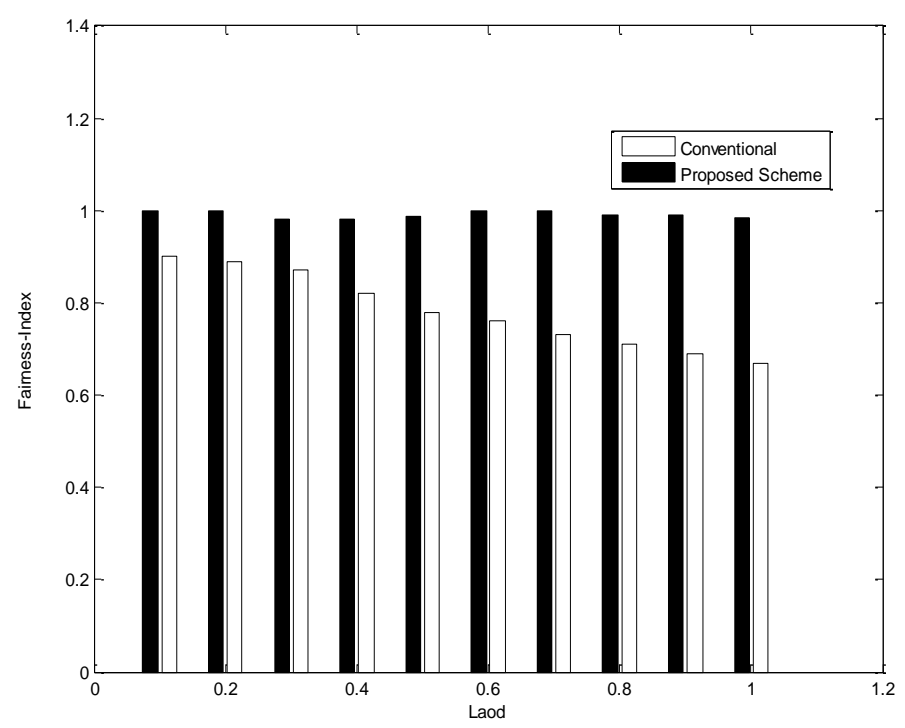

Figure 4: Variation in Fairness Index with respect to Load

Figure4 reveals that the proposed scheme can maintain acceptable throughput fairness amid all the flows for the entire load. As seen, the fairness-index of the proposed scheme is nearly unity; as a result it supports better QoS.

A new contention resolution scheme of the optical burst switching network based on deflection routing concept is analyzed in this article. An efficient implementation of CBDR scheme is presented. In the proposed technique, deflected burst travels through the least congested node to avoid the burst dropping and subsequent bursts are sent after adjusting the offset time to minimize the dropping of data bursts and to achieve acceptable level of fairness between traffic flows. The CBDR scheme enables the core nodes to monitor and manage the congestion in the optical network. Furthermore, the technique is uncomplicated, practical and its implementation does not lead to any compromise in the basic reason and main inspiration behind the emergence of the OBS technique, which is simplicity.

\section{CONCLUSION}

This paper proposes a novel congestion based scheme to reduce burst loss in OBS networks. Results have shown that the proposed CBDR scheme gives better outcome in case of burst dropping probability and throughput, but the end-to-end delay is on the higher side as compared to the conventional scheme under consideration. The proposed scheme avoids contention practically by controlling the burst sending rate according to blockage in the optical network. Consequently, the proposed technique can be employed where better drop ratio with better QoS is desirable amid feasible limits of delay.

Furthermore, it is clear that the functionality of CBDR scheme can be extended to provide flow and congestion control capabilities in the optical domain.

\section{REFERENCES}

[1] C. Qiao, M. Yoo, "Optical burst switching (OBS) - A new paradigm for an optical Internet," Journal of High Speed Networks, vol.8, pp. 69-84, 1999.

[2] Y. Chen, C. Qiao, "Performance Analysis of Optical Burst Switched Node with Deflection 
Routing," Proceedings of IEEE ICC, vol. 2, pp.1355-1359, 2003.

[3] Amit Gupta, R S Kaler, Harbhajan Singh, "Investigation of OBS Assembly Technique based on Various Scheduling Techniques for Maximizing Throughput", Optik - International Journal for Light and Electron Optics, Elsevier Science, In Press, Available Online, January 2012.

[4] Sungchan Kim, "Contention Resolution for Optical Burst Switching Networks Using Alternative Routing”, IEEE International Conference on Communications, vol.101, No.2, May 2002.

[5] Vinod M.Vokkarance, "Burst Segmentation:An Approach For Reducing Packet Loss In Optical Burst Switched Networks" IEEE International Conference on Communications, vol.101, No.1, May 2002.

[6] S Reza, M Hossain, S P Majumder, "Contention Problem in Optical Burst Switching Network", IEEE-International Conference on Computational Intelligence and Communication Networks", pp. 239-242, Nov 2010.

[7] A. Maach, G. V. Bochman and H. Mouftah, "Congestion Control and Contention Elimination in Optical Burst Switching," Telecommunication Systems, vol. 27, issue 2-4, pp. 115-131, Dec 2004.

[8] S. Y. Wang, "Using TCP Congestion Control to Improve the Performances of Optical Burst
Switched Networks," in Proc. IEEE ICC 2003, vol. 2, pp. 1438-1442, May 2003.

[9] F. Farahmand, Q. Zhang and J. P. Jue, "A ClosedLoop Rate-Based Contention Control for Optical Burst Switched Networks," in Proc. IEEE GLOBECOM 05, vol. 4, pp. 1989-1993, Dec 2005.

[10] Y. Chen, H. Wu, D. Xu, and C. Qiao, "Performance Analysis of Optical Burst Switched Node with Defection Routing", in IEEE ICC, vol. 2, pp. 1355-1359, 2003.

[11]A. Zalesky, "Evaluation of limited wavelength conversion and deflection routing as methods to reduce blocking probability in optical burst switched networks," Proceeding of IEEE ICC, vol.3, pp.1543- 1547, 2003.

[12] T. Coutelen, A. Metnani," "Measurement-based alternative routing strategies in optical burstswitched networks," Proceedings of 7th International Conference on Transparent Optical Networks, vol.1, pp.224- 227, 2005.

[13] T. C. K. Hui , C.K. Tham, "Adaptive Provisioning of Differentiated Services Networks based on Reinforcement Learning," IEEE Transactions on Systems, Man and Cybernetics, vol.4, pp.492$501,2003$.

[14] Amit Garg, R S Kaler, "An efficient routing scheme to reduce packet loss in all optical networks", Journal of Microwaves, Optoelectronics and Electromagnetic Applications, vol. 9, No 2, pp. 113-122, Dec 2010. 